\title{
Gauge theory of gravity on a four-dimensional covariant noncommutative space
}

\section{G. Manolakos*}

E-mail: giorgismanolakosegmail.com

\section{P. Manousselis}

National Technical University of Athens

E-mail: pman@central.ntua.gr

\section{G. Zoupanos}

National Technical University of Athens

E-mail: George.Zoupanos@cern.ch

\begin{abstract}
We review the construction of a four-dimensional noncommutative gravity model formulated in a gauge-theoretic way. For consistency, we include a brief review of gravity theories in four dimensions (General Relativity, Weyl Gravity), expressed as gauge theories. Also, we give a brief reminder of the formulation of gauge theories on noncommutative spaces. Last, the essential information of our recent work is included, specifically a gravity model constructed as a gauge theory on a four-dimensional covariant noncommutative space.
\end{abstract}

Corfu Summer Institute 2019 "School and Workshops on Elementary Particle Physics and Gravity" (CORFU2019)

31 August - 25 September 2019

Corfù, Greece

${ }^{*}$ Speaker. 


\section{Introduction}

If we consider that, at the regime of small distances (Planck length), the spatial coordinates exhibit a noncommutative behaviour, then the structure of the spacetime at this regime could be parametrized by the framework of noncommutative geometry. Referring now to higher distances (LHC scale) the Strong, Weak and Electromagnetic interactions are formulated by gauge theories and are described together in the Standard Model. At much smaller distances (but larger then Planck distances) the three interactions are studied in the interesting framework of Grand (Gauge) Unified Theories. Although none of the above two pictures include the gravitational interaction, there does exist a gauge-theoretic approach of it, see [1, 2, 3, 4, 5, 6, 7, 8, 9, 10,11, 12, 13], which is based on gauging the Lorentz and Poincaré groups in the pioneer works of Utiyama and Kibble $[1,2]$ and was extended by other authors $[4,5,6,7,8,9,10,11,12,13]$ gauging the de Sitter group, $S O(1,4)$, spontaneously broken by a scalar field to the Lorentz group, $S O(1,3)$. In this context the Weyl gravity has been constructed as a gauge theory of the four-dimensional conformal group [8, 9]. In the noncommutative framework in order to result with models of noncommutative gravity $[14,15,16,17,18,19,20,21,22]$ the above gauge-theoretic approach of gravity has been taken into consideration and translated in the noncommutative framework making use of the wellestablished formulation of gauge theories on noncommutative spaces.

In the above treatments the authors make use of the concepts of constant noncommutativity (Moyal-Weyl), the $\star$-product and the Seiberg-Witten map [23]. Besides the above approaches, construction of noncommutative gravitational models can be achieved using the noncommutative realization of matrix geometries [24, 25, 26, 27, 28, 29, 30]. Also, for alternatives see [31, 32, 33] (see also [34]). In general, it is understood that the formulation of noncommutative gravity is accompanied by the breaking of Lorentz invariance by noncommutative deformations. However, the existence of "covariant noncommutative spaces" [36, 37] which preserve the Lorentz invariance, allows the construction of field theories from noncommutative deformations. [38, 39, 40, 41, 42, $43,44,45,46,47]$ (see also [48, 49, 50, 51, 52]).

The purpose of this article is to present and highlight the features of a four-dimensional noncommutative gravity model, constructed recently [45] as a gauge theory on a fuzzy space, the $d S_{4}$. Drawing motivation from the work of Heckman-Verlinde [37], who were based on Yang's early publication [36], we have constructed a covariant fuzzy $d S$ space with the property of preserving Lorentz invariance. Requiring this property led to the enlargement of the isometry group of the fuzzy $d S_{4}$ from $S O(1,4)$ to $S O(1,5)$. Next, on the above (covariant) noncommutative space, the gauging of a subgroup of the total isometry took place, leading to the enlargement of the gauge group in a fixed representation. During the development of the gauge theory, the requirement of the covariance of the field strength tensor led to the addition of a 2-form gauge field. Eventually, our proposition of the action is of Yang-Mills type, including the kinetic term of the 2-form gauge field added earlier.

\section{Approaches of gravity as gauge theory}

\subsection{Gauge-theoretic approach of 4-d Einstein's Gravity}

Four-dimensional gravity is described, in a geometric way, by the successful Theory of Gen- 
eral Relativity. On the other hand, the gauge-theoretic alternative description of gravity aims, in principle, at a possible unification of the gravitational interaction with the rest ones, [1, 2, 4, 5, 6, 7]. In order to approach four-dimensional gravity in a gauge-theoretic way, first one has to employ the vierbein formulation of General Relativity. Then if a cosmological constant is present, according to its sign, the corresponding spaces on which the gauge theories are constructed are the Minkowski $M^{4}$, de Sitter $d S_{4}$ and anti-de-Sitter $A d S_{4}$, which are based on the Poincaré, $I S O(1,3)$, de Sitter, $S O(1,4)$, and Anti-de Sitter, $S O(2,3)$, gauge groups, respectively. The above isometry groups of the spaces are the indicated gauge groups in each case. Let us first recall the case in which cosmological constant is absent, that is the case of the Poincare group. In this case, the algebra is determined by the following commutation relations:

$$
\left[M_{a b}, M_{c d}\right]=4 \eta_{[a[c} M_{d] b]}, \quad\left[P_{a}, M_{b c}\right]=2 \eta_{a[b} P_{c]}, \quad\left[P_{a}, P_{b}\right]=0
$$

where $\eta_{a b}$ is the mostly positive metric tensor of the Minkowski spacetime and the generators $M_{a b}$ and $P_{a}$ correspond to the Lorentz transformations and the local translations, respectively. Then the gauge potential, $A_{\mu}$, is introduced which, by definition, takes values within the Poincaré algebra:

$$
A_{\mu}(x)=e_{\mu}{ }^{a}(x) P_{a}+\frac{1}{2} \omega_{\mu}^{a b}(x) M_{a b},
$$

where the gauge fields associated with the $P_{a}$ and $M_{a}$, i.e. $e_{\mu}{ }^{a}$ and $\omega_{\mu}{ }^{a b}$, are identified as the vierbein and spin connection, respectively. The gauge transformation of the connection $A_{\mu}$ is given by:

$$
\delta A_{\mu}=\partial_{\mu} \varepsilon+\left[A_{\mu}, \varepsilon\right],
$$

in which $\varepsilon=\varepsilon(x)$ is the parameter of the gauge transformation, which is an element of the algebra and therefore takes values in it:

$$
\varepsilon(x)=\xi^{a}(x) P_{a}+\frac{1}{2} \lambda^{a b}(x) M_{a b} .
$$

Combination of the equations (2.2) and (2.4) along with (2.3) produces the transformations of the gauge fields:

$$
\begin{aligned}
\delta e_{\mu}{ }^{a} & =\partial_{\mu} \xi^{a}+\omega_{\mu}{ }^{a b} \xi_{b}-\lambda^{a}{ }_{b} e_{\mu}{ }^{b}, \\
\delta \omega_{\mu}{ }^{a b} & =\partial_{\mu} \lambda^{a b}-2 \lambda^{[a}{ }_{c} \omega_{\mu}{ }^{c b]} .
\end{aligned}
$$

The standard procedure when building gauge theories continues with the definition of the corresponding field strength tensor:

$$
R_{\mu v}(A)=2 \partial_{[\mu} A_{v]}+\left[A_{\mu}, A_{v}\right]
$$

Being also valued in the algebra of generators it is written as an expansion on them:

$$
R_{\mu v}(A)=R_{\mu \nu}{ }^{a}(e) P_{a}+\frac{1}{2} R_{\mu \nu}{ }^{a b}(\omega) M_{a b}
$$

where $R_{\mu \nu}{ }^{a}$ and $R_{\mu \nu}{ }^{a b}$ are the component tensors, i.e. the curvatures corresponding to the component gauge fields, identified as the torsion and curvature, respectively. Their explicit forms are 
obtained after replacing equations (2.2) and (2.8) into the (2.7):

$$
\begin{aligned}
R_{\mu \nu}{ }^{a}(e) & =2 \partial_{[\mu} e_{v]}^{a}-2 \omega_{[\mu}^{a b} e_{v] b}, \\
R_{\mu \nu}{ }^{a b}(\omega) & =2 \partial_{[\mu} \omega_{v]}^{a b}-2 \omega_{[\mu}^{a c} \omega_{v] c}^{b} .
\end{aligned}
$$

Regarding the action of the theory, the indicated choice according to the standard procedure of building gauge theories is an action of Yang-Mills type, gauge invariant under $\operatorname{ISO}(1,3)$ transformations. However, this is not the case since, such a choice, would lead to an action not identical to the Einstein-Hilbert, which we already know that is the desired one. For this reason, less straightforward route is necessary to be followed. Along these lines, instead of the Poincare group, it is rather more convenient to gauge the $\mathrm{SO}(1,4)$ group and incorporate a spontaneous symmetry breaking mechanism induced by the presence of a scalar field in the fundamental representation $[4,6]$.

For this specific purpose, the alternative choice of the four-dimensional de Sitter group is preferred to the one of the Poincare because, although the number of the generators is equal in two cases, in the de Sitter case the generators are distributed on equal footing. In this context, the spontaneous symmetry breaking due to the scalar field leads to the breaking of the part associated to the translations and results to a more constrained theory involving the Ricci scalar along with a Gauss-Bonnet term with vanishing torsion. The resulting action of the theory is Lorentz invariant and is actually the Einstein-Hilbert action.

In a nutshell, as far as the transformations of the fields and the calculation of the tensors is concerned, Einstein's gravity can be described as a gauge theory of the Poincaré group. In turn, the dynamical part, that is the action of the theory, is obtained by making us of the de Sitter group instead along with an extra scalar field, the presence of which induces appropriate spontaneous breaking which leads to the correct Einstein-Hilbert action.

\subsection{Gauge-theoretic approach of Weyl gravity}

Another successful translation of a gravity theory into the gauge-theoretic context is that of Weyl gravity theory. In this case, the group to be gauged is the four-dimensional conformal group, that is $\operatorname{SO}(2,4)$. The transformations of the fields and the curvature tensors (derived from the field strength tensor) are obtained straightforwardly. The gauge invariant action that is considered is of Yang-Mills type and is broken by imposing particular conditions on the curvature tensors. Imposition of these constraints recover the desired scale invariant Weyl action $[8,9,10]$ (see also $[11,12])$.

The generators of the conformal algebra of $\mathrm{SO}(2,4)$ comprises of the local translations $\left(P_{a}\right)$, the Lorentz transformations $\left(M_{a b}\right)$, the conformal boosts $\left(K_{a}\right)$ and the dilatations $(D)$. The algebra they satisfy is determined by the following commutation relations:

$$
\begin{gathered}
{\left[M_{a b}, M^{c d}\right]=4 M_{[a}^{[d} \delta_{b]}^{c]}, \quad\left[M_{a b}, P_{c}\right]=2 P_{[a} \delta_{b] c}, \quad\left[M_{a b}, K_{c}\right]=2 K_{[a} \delta_{b] c}} \\
{\left[P_{a}, D\right]=P_{a}, \quad\left[K_{a}, D\right]=-K_{a}, \quad\left[P_{a}, K_{b}\right]=2\left(\delta_{a b} D-M_{a b}\right),}
\end{gathered}
$$

where $a, b, c, d=1 \ldots 4$. The gauging procedure dictates that the gauge potential, $A_{\mu}$, of the theory is written down as expansion on the algebra generators:

$$
A_{\mu}=e_{\mu}^{a} P_{a}+\frac{1}{2} \omega_{\mu}^{a b} M_{a b}+b_{\mu} D+f_{\mu}^{a} K_{a}
$$


in which it is evident that each generator admits a gauge field. The vierbein and the spin connection are identified as gauge fields of the theory, as they were in the previous case of Einstein gravity. The gauge potential, (2.12), is transformed under the following rule:

$$
\delta_{\varepsilon} A_{\mu}=D_{\mu} \varepsilon=\partial_{\mu} \varepsilon+\left[A_{\mu}, \varepsilon\right],
$$

in which the inclusion of a gauge transformation parameter has taken place. The parameter is valued in the Lie algebra of the $\mathrm{SO}(2,4)$, therefore it is written down in the following expansion form:

$$
\varepsilon=\varepsilon_{P}^{a} P_{a}+\frac{1}{2} \varepsilon_{M}^{a b} M_{a b}+\varepsilon_{D} D+\varepsilon_{K}^{a} K_{a} .
$$

The transformations of the various gauge fields is obtained by the combination of equations (2.13), (2.12) and (2.14):

$$
\begin{aligned}
\delta e_{\mu}^{a} & =\partial_{\mu} \varepsilon_{P}^{a}+2 i e_{\mu b} \varepsilon_{M}^{a b}-i \omega_{\mu}^{a b} \varepsilon_{P b}-b_{\mu} \varepsilon_{K}^{a}+f_{\mu}^{a} \varepsilon_{D}, \\
\delta \omega_{\mu}^{a b} & =\frac{1}{2} \partial_{\mu} \varepsilon_{M}^{a b}+4 i e_{\mu}{ }_{\mu} \varepsilon_{P}^{b}+\frac{i}{4} \omega_{\mu}^{a c} \varepsilon_{M c}^{b}+i f_{\mu}^{a} \varepsilon_{K}^{b}, \\
\delta b_{\mu} & =\partial_{\mu} \varepsilon_{D}-e_{\mu}^{a} \varepsilon_{K a}+f_{\mu}^{a} \varepsilon_{P a}, \\
\delta f_{\mu}^{a} & =\partial_{\mu} \varepsilon_{K}^{a}+4 i e_{\mu}^{a} \varepsilon_{D}-i \omega_{\mu}^{a b} \varepsilon_{K b}-4 i b_{\mu} \varepsilon_{P}^{a}+i f_{\mu}^{b} \varepsilon_{M b}^{a} .
\end{aligned}
$$

In turn, the field strength tensor is determined by the relation:

$$
R_{\mu v}=2 \partial_{[\mu} A_{v]}-i\left[A_{\mu}, A_{v}\right]
$$

and is also expanded on the various generators:

$$
R_{\mu v}=\tilde{R}_{\mu v}^{a} P_{a}+\frac{1}{2} R_{\mu \nu}^{a b} M_{a b}+R_{\mu v}+R_{\mu \nu}^{a} K_{a} .
$$

Then putting together equations (2.16) and (2.17) lead to the following expressions of the various component curvature tensors:

$$
\begin{aligned}
& R_{\mu \nu}{ }^{a}(P)=2 \partial_{[\mu} e_{v]}^{a}+f_{[\mu}^{a} b_{v]}+e_{[\mu}^{b} \omega_{v]}^{a c} \delta_{b c}, \\
& R_{\mu v}{ }^{a b}(M)=\partial_{[\mu} \omega_{v]}^{a b}+\omega_{[\mu}^{c a} \omega_{v]}^{d b} \delta_{c d}+e_{[\mu}^{a} e_{v]}^{b}+f_{[\mu}^{a} f_{v]}^{b} \text {, } \\
& R_{\mu v}(D)=2 \partial_{[\mu} b_{v]}+f_{[\mu}{ }^{a} e_{v]}^{b} \delta_{a b}, \\
& R_{\mu \nu}{ }^{a}(K)=2 \partial_{[\mu} f_{v]}^{a}+e_{[\mu}^{a} b_{v]}+f_{[\mu}^{b} \omega_{v]}^{a c} \delta_{b c} .
\end{aligned}
$$

The choice of the action of the theory is of Yang-Mills type that is gauge invariant under the $\mathrm{SO}(2,4)$ symmetry. This symmetry breaks after the imposition of specific constraints $[8,9,10]$, specifically the torsionless condition, $R(P)=0$ along with an additional constraint on the $R(M)$ tensor. If these constraints are solved algebraically, they yield expressions of the gauge fields $\omega_{\mu}{ }^{a b}$ and $f_{\mu}{ }^{a}$ in terms of the rest independent fields $e_{\mu}{ }^{a}$ and $b_{\mu}$. Furthermore, the field $b_{\mu}$ can be fixed to the specific gauge of $b_{\mu}=0$. Therefore, taking all the constraints into account, the initial action takes the form of the well-known Weyl action, which is invariant under diffeomorphisms and scale transformations ${ }^{1}$.

\footnotetext{
${ }^{1}$ Besides the above breaking of the conformal symmetry, there exists another breaking route via constraints [46], which leads to a Lorentz invariant action, more explicitly the Einstein-Hilbert action.
} 
From our point of view, such an outcome could be produced after employing an alternative symmetry breaking mechanism, induced by two scalar fields in the fundamental representation of $\mathrm{SO}(2,4)[47]$. The spontaneous symmetry breaking could be viewed as a generalization of the case of the de Sitter group breaking down to the Lorentz group by a single scalar in the fundamental representation of $\mathrm{SO}(1,4)$ (see previous section 2.1).

\section{Gauge theories on noncommutative spaces}

In this section we include the basics regarding the construction of gauge theories on noncommutative spaces, since it is fundamental for our purposes.

In noncommutative geometry, gauge fields arise in a very natural way and are intertwined with the notion of covariant coordinate [53], that is the noncommutative analogue of the covariant derivative as we will stress later.

Let us now begin with considering a field $\phi\left(X_{a}\right)$ on a fuzzy space, depending on the noncommuting coordinates $X_{a}$. The field belongs to a representation of a gauge group $G$, therefore an infinitesimal gauge transformation $\delta \phi$ with gauge transformation parameter $\lambda\left(X_{a}\right)$ is given by:

$$
\delta \phi(X)=\lambda(X) \phi(X)
$$

In case the transformation parameter $\lambda(X)$ is simply a function of the coordinates, $X_{a}$, then it is considered as an infinitesimal Abelian transformation and the gauge group is $G=U(1)$, while in case $\lambda(X)$ is a $P \times P$ matrix, then it can be viewed as a gauge transformation of the non-Abelian gauge group $G=U(P)$, i.e. the group including all hermitian $P \times P$ matrices. It is worth-noting that the coordinates are invariant under transformations of the gauge group, $G$, that is $\delta X_{a}=0$. In turn, let us perform a gauge transformation on the product of a coordinate and the field:

$$
\delta\left(X_{a} \phi\right)=X_{a} \lambda(X) \phi
$$

The above transformation is not a covariant one since, in general, it holds:

$$
X_{a} \lambda(X) \phi \neq \lambda(X) X_{a} \phi .
$$

Drawing ideas from the methodology of ordinary gauge theories, in which covariant derivative is defined for similar reasons, in the noncommutative case, the covariant coordinate, $\phi_{a}$, is introduced by its transformation rule:

$$
\delta\left(\phi_{a} \phi\right)=\lambda \phi_{a} \phi,
$$

which is satisfied in case that:

$$
\delta\left(\phi_{a}\right)=\left[\lambda, \phi_{a}\right] .
$$

Eventually, the covariant coordinate is defined as:

$$
\phi_{a} \equiv X_{a}+A_{a},
$$

where it is straightforward to identify $A_{a}$ as the gauge connection of the theory. Putting together equations (3.5), (3.6), the gauge transformation of the connection, $A_{a}$, is obtained:

$$
\delta A_{a}=-\left[X_{a}, \lambda\right]+\left[\lambda, A_{a}\right],
$$


giving an a posteriori explanation of the interpretation of $A_{a}$ as a gauge field ${ }^{2}$. Accordingly, the corresponding field strength tensor, $F_{a b}$, is defined as follows:

$$
F_{a b} \equiv\left[X_{a}, A_{b}\right]-\left[X_{b}, A_{a}\right]+\left[A_{a}, A_{b}\right]-C_{a b}^{c} A_{c}=\left[\phi_{a}, \phi_{b}\right]-C_{a b}^{c} \phi_{c}
$$

which is easily proven to be covariant under a gauge transformation,

$$
\delta F_{a b}=\left[\lambda, F_{a b}\right] .
$$

The above scheme will be used in the following sections in the construction of gravity model as gauge theory on fuzzy covariant space.

\section{Four-dimensional noncommutative gravity}

We proceed with the construction of a model of four-dimensional noncommutative gravity as a gauge theory on a covariant fuzzy space. First, we need to present the four-dimensional fuzzy space we defined in order to build the theory on it.

\subsection{Four-dimensional fuzzy covariant spaces of $d S_{4}$ and $S^{4}$}

Here we construct the fuzzy version of four-dimensional de Sitter space, $d S_{4}$, as it is the one we use as the background space in our model. The continuous $d S_{4}$ can be be defined as an embedding in the five-dimensional Minkowski space:

$$
\eta^{M N} x_{M} x_{N}=R^{2}
$$

$M, N=0, \ldots, 4$ and $\eta^{M N}$ is the metric tensor of the five-dimensional Minkowski spacetime, $\eta^{M N}=$ $\operatorname{diag}(-1,+1,+1,+1,+1)$. The construction of its fuzzy analogue is achieved if the coordinates, $X_{m}$, are considered, instead of numbers, to be operators that fail to commute with each other:

$$
\left[X_{m}, X_{n}\right]=i \theta_{m n}
$$

where the spacetime indices are $m, n=1, \ldots, 4$. Drawing lessons from the well-known fuzzy sphere case $[24,25]$, in which the corresponding coordinates-operators are corresponded to the (rescaled) three generators of $S U(2)$ assigned in an N-dimensional representation, the right hand side in eq(4.2), has to be identified with a generator of the underlying algebra. Such an identification ensures covariance, i.e $\theta_{m n}=C_{m n}{ }^{r} X_{r}$, with $C_{m n r}$ being a rescaled Levi-Civita symbol. Identified otherwise, if for instance the right hand side in eq(4.2) was fixed to an antisymmetric tensor, then the Lorentz invariance would be violated. However, in the fuzzy de Sitter case in which we are interested, such an identification cannot be achieved, since the algebra would not be closing [37] ${ }^{3}$. Since covariance is an indispensable property of the space we are building, a promising suggestion $[36,37]$ is to make use of a group of larger symmetry, in which all generators and the noncommutativity would be included in it. Targeting minimal extension of the symmetry, we are led to adopt

\footnotetext{
${ }^{2}$ For more details see [34]

${ }^{3}$ For more information on this issue, see $[49,50]$, where the same problem is encountered in their construction of the fuzzy four-sphere.
} 
the $S O(1,5)$ group. Therefore, a fuzzy version of $d S_{4}$ space, respecting covariance, is obtained after the symmetry is enlarged to the $S O(1,5)$ [45]. To facilitate the whole construction and the calculations we adopt the Euclidean signature, which means that instead of the $S O(1,5)$, the symmetry group we consider is that of $S O(6)$. Thus we have resulted with the Euclidean version of the covariant fuzzy $d S_{4}$, that is a covariant fuzzy $S^{4}$. To describe this space an approach similar to the fuzzy sphere is possible, specifically we will make use of the approximation by finite-dimensional matrices.

Explicit formulation of the above fuzzy space is performed by considering the $S O(6)$ (antisymmetric) generators, $\mathrm{J}_{A B}$, with $A, B=1, \ldots, 6$, which obey the following commutation relation:

$$
\left[J_{A B}, J_{C D}\right]=i\left(\delta_{A C} J_{B D}+\delta_{B D} J_{A C}-\delta_{B C} J_{A D}-\delta_{A D} J_{B C}\right)
$$

Writing down these generators as decompositions in $S O(4)$ notation $^{4}$, the various component generators are identified as follows:

$$
J_{m n}=\frac{1}{\hbar} \Theta_{m n}, \quad J_{m 5}=\frac{1}{\lambda} X_{m}, J_{m 6}=\frac{\lambda}{2 \hbar} P_{m}, J_{56}=\frac{1}{2} \mathrm{~h},
$$

where $m, n=1, \ldots, 4$. For reasons of correct dimensionality, an elementary length, $\lambda$, has been introduced in the above identifications. The coordinates and momenta operators as well as the noncommutativity tensor are denoted as $X_{m}, P_{m}$ and $\Theta_{m n}$, respectively, satisfying the following commutation relations:

$$
\begin{gathered}
{\left[X_{m}, X_{n}\right]=i \frac{\lambda^{2}}{\hbar} \Theta_{m n}, \quad\left[P_{m}, P_{n}\right]=4 i \frac{\hbar}{\lambda^{2}} \Theta_{m n},} \\
{\left[X_{m}, P_{n}\right]=i \hbar \delta_{m n} \mathrm{~h}, \quad\left[X_{m}, \mathrm{~h}\right]=i \frac{\lambda^{2}}{\hbar} P_{m},} \\
{\left[P_{m}, \mathrm{~h}\right]=4 i \frac{\hbar}{\lambda^{2}} X_{m},} \\
{\left[X_{m}, \Theta_{n p}\right]=i \hbar\left(\delta_{m p} X_{n}-\delta_{m n} X_{p}\right)} \\
{\left[P_{m}, \Theta_{n p}\right]=i \hbar\left(\delta_{m p} P_{n}-\delta_{m n} P_{p}\right)} \\
{\left[\Theta_{m n}, \Theta_{p q}\right]=i \hbar\left(\delta_{m p} \Theta_{n q}+\delta_{n q} \Theta_{m p}-\delta_{n p} \Theta_{m q}-\delta_{m q} \Theta_{n p}\right)} \\
{\left[\mathrm{h}, \Theta_{m n}\right]=0 .}
\end{gathered}
$$

It is notable to refer that the above algebra, in contrast to the Heisenberg algebra, admits finitedimensional matrices representing the operators $X_{m}, P_{m}$ and $\Theta_{m n}$ and therefore the spacetime obtained above is considered to be a finite quantum system [37, 51, 54].

\subsection{Gravity as gauge theory on the four-dimensional fuzzy covariant space}

In the procedure of the construction of Einstein gravity as gauge theory (section 2.1), in which the isometry group (the Poincaré group) was the one that was gauged, accordingly in this case the gauge group is the isometry group of the Euclidian version of the fuzzy $d S_{4}$ space, i.e. the $S O(5)$,

\footnotetext{
${ }^{4}$ The embedding path we consider is $S O(6) \supset S O(5) \supset S O(4)$.
} 
viewed as a subgroup of the $S O(6)$ group. In general, in noncommutative gauge theories, anticommutators of the generators of the algebra play a key role, as we have already explained in detail in our previous works [38, 39] (see also [17]). Specifically in the current case, the anticommutation relations of the generators of the gauge group, $S O(5)$, produce operators that, in principle, are not elements of the algebra. The treatment to this pathology is to fix the representation in which the generators are assigned and then include all operators produced by the anticommutators of the generators into the algebra, extending the initial Lie algebra. Following the above reasoning, we are eventually led to extend $S O(5)$ to $S O(6) \times U(1)(\sim U(4))$, with the generators being $4 \times 4$ matrices in the spinor representation of $S O(6)$ (or the fundamental of $S U(4)), 4$.

Aiming at the specific expressions of the matrices representing the generators, the Euclidean $\Gamma$-matrices are employed, satisfying the following well-known anticommutation relation:

$$
\left\{\Gamma_{a}, \Gamma_{b}\right\}=2 \delta_{a b} \mathbf{1},
$$

where $a, b=1, \ldots, 4$. The $\Gamma_{5}$ matrix is defined as $\Gamma_{5}=\Gamma_{1} \Gamma_{2} \Gamma_{3} \Gamma_{4}$. Therefore, it is deduced that the generators of the $\mathrm{SO}(6) \times \mathrm{U}(1)$ gauge group are identified as the following matrices:

a) Six generators of the Lorentz transformations: $\mathrm{M}_{a b}=-\frac{i}{4}\left[\Gamma_{a}, \Gamma_{b}\right]=-\frac{i}{2} \Gamma_{a} \Gamma_{b}, a<b$,

b) four generators of the conformal boosts: $\mathrm{K}_{a}=\frac{1}{2} \Gamma_{a}$,

c) four generators of the local translations: $\mathrm{P}_{a}=-\frac{i}{2} \Gamma_{a} \Gamma_{5}$,

d) one generator for special conformal transformations: $\mathrm{D}=-\frac{1}{2} \Gamma_{5}$ and

e) one U(1) generator: 1 .

The form of the $\Gamma$-matrices is determined by tensor products of the Pauli matrices:

$$
\begin{gathered}
\Gamma_{1}=\sigma_{1} \otimes \sigma_{1}, \quad \Gamma_{2}=\sigma_{1} \otimes \sigma_{2}, \quad \Gamma_{3}=\sigma_{1} \otimes \sigma_{3} \\
\Gamma_{4}=\sigma_{2} \otimes \mathbf{1}, \quad \Gamma_{5}=\sigma_{3} \otimes \mathbf{1} .
\end{gathered}
$$

It is straightforward to calculate the commutation relations of the various operators:

$$
\begin{aligned}
{\left[K_{a}, K_{b}\right] } & =i M_{a b}, \quad\left[P_{a}, P_{b}\right]=i M_{a b} \\
{\left[X_{a}, P_{b}\right] } & =i \delta_{a b} D, \quad\left[X_{a}, D\right]=i P_{a} \\
{\left[P_{a}, D\right] } & =i K_{a}, \quad\left[K_{a}, P_{b}\right]=i \delta_{a b} D, \quad\left[K_{a}, D\right]=-i P_{a} \\
{\left[K_{a}, M_{b c}\right] } & =i\left(\delta_{a c} K_{b}-\delta_{a b} K_{c}\right) \\
{\left[P_{a}, M_{b c}\right] } & =i\left(\delta_{a c} P_{b}-\delta_{a b} P_{c}\right) \\
{\left[M_{a b}, M_{c d}\right] } & =i\left(\delta_{a c} M_{b d}+\delta_{b d} M_{a c}-\delta_{b c} M_{a d}-\delta_{a d} M_{b c}\right) \\
{\left[D, M_{a b}\right] } & =0 .
\end{aligned}
$$

After the determination of the commutation relations of the generators, the process of building a noncommutative gauge theory can be performed in a straightforward way. First, the covariant 
coordinate of the theory is defined as:

$$
\hat{X}_{m}=X_{m} \otimes \mathbf{1}+A_{m}(X) .
$$

The coordinate $\hat{X}_{m}$ is covariant by construction and this property is expressed as:

$$
\delta \hat{X}_{m}=i\left[\varepsilon, \hat{X}_{m}\right]
$$

where $\varepsilon(X)$ is the transformation parameter of the gauge theory, which is a function of the noncommutative coordinates $\left(N \times N\right.$ matrices), $X_{m}$, but, at the same time, it is valued in the $S O(6) \times U(1)$ algebra. Therefore, it can be written as a decomposition on the sixteen generators of the algebra:

$$
\varepsilon=\varepsilon_{0}(X) \otimes \mathbf{1}+\xi^{a}(X) \otimes K_{a}+\tilde{\varepsilon}_{0}(X) \otimes D+\lambda_{a b}(X) \otimes \Sigma^{a b}+\tilde{\xi}^{a}(X) \otimes P_{a} .
$$

Combination of the equations (4.14), (4.15) and (4.16) yields the transformation rule of $A_{m}$, since it holds that $\delta X_{m}=0$. In accordance with the commutative case, the transformation rule of $A_{m}$ implies that it can be interpreted as the connection of the gauge theory.

The connection $A_{m}$ follows the same pattern with the $\varepsilon$, that is taking values in the $S O(6) \times$ $U(1)$ algebra, besides that it is a function of the coordinates $X_{m}$ of the fuzzy space $d S_{4}$. This leads to the following relation:

$$
\begin{aligned}
A_{m}(X)=e_{m}^{a}(X) \otimes & P_{a}+\omega_{m}^{a b}(X) \otimes \Sigma_{a b}(X)+b_{m}^{a}(X) \otimes K_{a}(X) \\
& +\tilde{a}_{m}(X) \otimes D+a_{m}(X) \otimes \mathbf{1},
\end{aligned}
$$

in which various gauge fields have been introduced for every generator of the algebra of $S O(6) \times$ $U(1)$. The component gauge fields that were introduced above are functions of the space coordinates, $X_{m}$, meaning that they have the form of $N \times N$ matrices, where $N$ is the dimension of the representation of the coordinates. In turn, among the gauge fields and their corresponding generators, the tensor product is used, because of the fact that the factors are matrices of different dimensions, since generators are represented by $4 \times 4$ matrices. Concluding the reasoning, all terms of the expression of the gauge connection are $4 N \times 4 N$ matrices.

In turn, the covariant coordinate is also given as:

$$
\hat{X}_{m}=X_{m} \otimes \mathbf{1}+e_{m}^{a}(X) \otimes P_{a}+\omega_{m}^{a b}(X) \otimes \Sigma_{a b}+b_{m}^{a} \otimes K_{a}+\tilde{a}_{m} \otimes D+a_{m} \otimes \mathbf{1} .
$$

The next step is to calculate the field strength tensor of the gauge theory we are building. In our case, the field strength tensor is defined as:

$$
\mathscr{R}_{m n}=\left[\hat{X}_{m}, \hat{X}_{n}\right]-\frac{i \lambda^{2}}{\hbar} \hat{\Theta}_{m n}
$$

in which $\hat{\Theta}_{m n}=\Theta_{m n} \otimes \mathbf{1}+\mathscr{B}_{m n}$. The $\mathscr{B}_{m n}$ is a 2-form gauge field, valued in the algebra of $\mathrm{SO}(6) \times \mathrm{U}(1)$. The introduction of $\mathscr{B}_{m n}$ took place in order to make the field strength tensor covariant ${ }^{5}$. The presence of $\mathscr{B}_{m n}$ field affects the form of the action of the theory, with an addition of a kinetic term of the form:

$$
\mathscr{S}_{\mathscr{B}}=\operatorname{Tr} \operatorname{tr} \hat{\mathscr{H}}_{\text {mnp }} \hat{\mathscr{H}}^{\text {mnp }}
$$

\footnotetext{
${ }^{5}$ More information on this issue is given in Appendix A of the original paper of [45].
} 
The $\hat{\mathscr{H}}_{\text {mn }}$ field strengthis a covariant tensor, therefore the above action is invariant under a gauge transformation.

Belonging in the underlying algebra, the field strength tensor of the gauge connection, (4.19), can be expanded in terms of the component curvature tensors:

$$
\begin{gathered}
\mathscr{R}_{m n}(X)=R_{m n}{ }^{a b}(X) \otimes \Sigma_{a b}+\tilde{R}_{m n}^{a}(X) \otimes P_{a}+R_{m n}^{a}(X) \otimes K_{a} \\
+\tilde{R}_{m n}(X) \otimes D+R_{m n}(X) \otimes \mathbf{1} .
\end{gathered}
$$

Having defined all necessary elements, the transformations of the gauge fields and the component curvature tensors can now be obtained. The explicit expressions and calculations can be found in the first paper of ref.[45].

\subsection{The action and symmetry breaking by constraints}

Let us now focus on the dynamics of the theory determining the action. In our case it is natural to consider one of Yang-Mills type ${ }^{6}$ :

$$
\mathscr{S}=\operatorname{Tr} \operatorname{tr}\left\{\mathscr{R}_{m n}, \mathscr{R}_{r s}\right\} \varepsilon^{m n r s},
$$

where $\operatorname{Tr}$ denotes the trace over the coordinates which are $N \times N$ matrices and stands in the place of integration. The tr denotes the trace over the generators of the algebra.

Nevertheless, the desired action of the theory is that of Lorentz, in Euclidean signature, the $S O(4)$. A brute force way to achieve this is to render every tensor not related to the Lorentz subgroup as vanishing, with minor exception of the $U(1)$ tensor. This way, a breaking of the initial $S O(6) \times U(1)$ symmetry to the $S O(4) \times U(1)$ is achieved. However, simply by counting the degrees of freedom, adoption of the above breaking would lead to an overconstrained theory. For this reason, we perform the symmetry breaking imposing less straightforward constraints [45]. In turn, the first condition is the torsionless condition:

$$
\tilde{R}_{m n}^{a}(P)=0
$$

which is also encountered in the cases of the Einstein and conformal gravity theories when described as gauge theories. Now, the fact that the gauge field $b_{m}^{a}$ can be viewed as a second vielbein of the theory, would render the theory as bimetric, which is not what we desire in the this case. Therefore, in order to avoid the bimetric interpretation, it is preferable to consider $e_{m}^{a}=b_{m}^{a}$ when solving the constraint. This choice leads to an expression of the spin connection $\omega_{m}^{a b}$ in terms of the independent fields, $e_{m}^{a}, a_{m}, \tilde{a}_{m}$. The explicit expression of the spin connection in terms of the other fields is obtained after employing the following two identities:

$$
\delta_{f g h}^{a b c}=\varepsilon^{a b c d} \varepsilon_{f g h d} \quad \text { and } \quad \frac{1}{3 !} \delta_{f g h}^{a b c} a^{f g h}=a^{[f g h]} .
$$

Solution of the constraint $\tilde{R}(P)=0$ leads to:

$$
\varepsilon^{a b c d}\left[e_{m}^{b}, \omega_{n}^{c d}\right]-i\left\{\omega_{m}^{a b}, e_{n b}\right\}=-\left[D_{m}, e_{m}^{a}\right]-i\left\{e_{m}^{a}, \tilde{a}_{m}\right\}
$$

\footnotetext{
${ }^{6} \mathrm{~A}$ Yang-Mills action of a theory on the fuzzy $d S_{4}$ space is gauge invariant. For details see Appendix A of the first paper of [45].
} 
where $D_{m}=X_{m}+a_{m}$, that is the covariant coordinate of an Abelian noncommutative gauge theory. The above equation leads to the following two:

$$
\varepsilon^{a b c d}\left[e_{m}^{b}, \omega_{n}^{c d}\right]=-\left[D_{m}, e_{m}^{a}\right] \quad \text { and } \quad\left\{\omega_{m}^{a b}, e_{n b}\right\}=\left\{e_{m}^{a}, \tilde{a}_{n}\right\}
$$

The expression of the spin connection in terms of the independent fields is given after taking into account the identities, (4.24):

$$
\omega_{n}^{a c}=-\frac{3}{4} e_{b}^{m}\left(-\varepsilon^{a b c d}\left[D_{m}, e_{n d}\right]+\delta^{[b c}\left\{e_{n}^{a]}, \tilde{a}_{m}\right\}\right) .
$$

According to [55], one could make use of the argument that the vanishing of the field strength tensor in a gauge theory can lead to the vanishing of the corresponding gauge field. However, in the case of $\tilde{R}(P)=0$ we do not apply the above argument, $e_{\mu}^{a}=0$, because this choice would lead to a degenerate metric tensor of the space [13]. On the other hand, the field that can be fixed in a gauge in which it is set to zero is the $\tilde{a}_{m}$. This fixing, $\tilde{a}_{m}=0$, will, in turn, modify the expression of the spin connection, (4.27), producing an even more simplified expression of the spin connection in terms of the vielbein:

$$
\omega_{n}^{a c}=\frac{3}{4} e_{b}^{m} \varepsilon^{a b c d}\left[D_{m}, e_{n d}\right] .
$$

It should be noted that the $R_{m n}(\mathbf{1})$ tensor, related to the noncommutativity of the space, is not considered as vanishing. The $U(1)$ part of the initial theory remains unbroken in the resulting theory after the breaking as it is still a theory on a noncommutative space. However, the corresponding field, $a_{m}$, will vanish in case the commutative limit of the broken theory is considered. In this limit, the noncommutativity is relieved, $a_{m}$ decouples being superheavy and also the gauge theory is just $S O(4)$. An alternative way to break the $S O(6)$ gauge symmetry down to the desired $S O(4)$ would be achieved with the induction of a spontaneous symmetry breaking by including in the theory two extra scalar fields in the 6 representation of $S O(6)$ [47], translating the argument developed in the case of conformal gravity to the noncommutative framework. Our expectation is that the spontaneous symmetry breaking induced by the scalars would also lead to a constrained theory as the above by the imposition of the constraints (4.23).

Now, after the symmetry breaking, i.e. including the constraints, the action will be:

$$
\begin{aligned}
& \mathscr{S}=2 \operatorname{Tr}\left(R_{m n}{ }^{a b} R_{r s}{ }^{c d} \varepsilon_{a b c d} \varepsilon^{m n r s}+4 \tilde{R}_{m n} R_{r s} \varepsilon^{m n r s}\right. \\
&+\left.\frac{1}{3} H_{m n p}{ }^{a b} H^{m n p c d} \varepsilon_{a b c d}+\frac{4}{3} \tilde{H}_{m n p} H^{m n p}\right) .
\end{aligned}
$$

Eventually, writing down the explicit expressions of the component tensors as well as the $\omega$ gauge field in terms of the non-vanishing gauge fields, (4.28) and then varying with respect to the independent gauge fields would produce the equations of motion.

\section{Conclusions}

In this review we presented a four-dimensional gravity model as a gauge theory on a fuzzy version of the (Euclidian version) four-dimensional de Sitter space. The constructed fuzzy space, $d S_{4}$, consists a four-dimensional covariant noncommutative space. In turn, although the initial gauge 
group was the isometry group of $d S_{4}$, that is $S O(1,4)$, we were led to extend it to $S O(1,5) \times U(1)$ for the sake of the inclusion of the anticommutators of the generators in a fixed respresentation. Then, in accordance to the standard procedure, we moved on by calculating the transformations of the fields and the component curvature tensors. Since we were aiming to result with a theory respecting the $S O(1,3)$ "Lorentz" symmetry, we were led to impose certain conditions (constraints) in order to break the symmetry of the initial gauge group. After the breaking, including the solutions of the constraints, the action takes its final form and with variation the equations of motion are obtained. The latter will be included in future work. It should be noted that, at the point before the application of the symmetry breaking, the expressions of the nnoncommutative gauge theory we constructed reduce to the ones of the conformal gravity in the commutative limit. Last, it should be also highlighted that the above is a matrix model which gives insight into the gravitational interaction in the high-energy regime where noncommutativity can be considered and also giving promises for improved UV properties as compared to ordinary gravity. The latter, as well as the inclusion of matter fields is a subject of further study.

Acknowledgements:

We would like to thank Ali Chamseddine, Paolo Aschieri, Thanassis Chatzistavrakidis, Evgeny Ivanov, Larisa Jonke, Danijel Jurman, Alexander Kehagias, Dieter Lust, Denjoe O'Connor, Emmanuel Saridakis, Harold Steinacker, Kelly Stelle, Patrizia Vitale and Christof Wetterich for useful discussions. One of us (GZ) has been supported within the Excellence Initiative funded by the German and States Governments, at the Institute for Theoretical Physics, Heidelberg University and from the Excellent Grant Enigmass of LAPTh. GZ would like to thank the ITP - Heidelberg, LAPTh - Annecy and MPI - Munich for their hospitality.

\section{References}

[1] R. Utiyama, "Invariant theoretical interpretation of interaction," Phys. Rev. 101 (1956) 1597. doi:10.1103/PhysRev.101.1597

[2] T. W. B. Kibble, "Lorentz invariance and the gravitational field," J. Math. Phys. 2 (1961) 212. doi: $10.1063 / 1.1703702$

[3] F. W. Hehl, P. Von Der Heyde, G. D. Kerlick and J. M. Nester, "General Relativity with Spin and Torsion: Foundations and Prospects," Rev. Mod. Phys. 48 (1976) 393. doi:10.1103/RevModPhys.48.393

[4] K. S. Stelle and P. C. West, "Spontaneously Broken De Sitter Symmetry and the Gravitational Holonomy Group," Phys. Rev. D 21 (1980) 1466. doi:10.1103/PhysRevD.21.1466

[5] S. W. MacDowell and F. Mansouri, "Unified Geometric Theory of Gravity and Supergravity," Phys. Rev. Lett. 38 (1977) 739 Erratum: [Phys. Rev. Lett. 38 (1977) 1376]. doi:10.1103/PhysRevLett.38.1376, 10.1103/PhysRevLett.38.739

[6] E. A. Ivanov and J. Niederle, Conference: C80-06-23.3, p.545-551, 1980; "On Gauge Formulations Of Gravitation Theories.," E. A. Ivanov and J. Niederle, "Gauge Formulation of Gravitation Theories. 1. The Poincare, De Sitter and Conformal Cases," Phys. Rev. D 25 (1982) 976. doi:10.1103/PhysRevD.25.976; E. A. Ivanov and J. Niederle, "Gauge Formulation of Gravitation Theories. 2. The Special Conformal Case," Phys. Rev. D 25 (1982) 988. doi:10.1103/PhysRevD.25.988 
[7] T. W. B. Kibble and K. S. Stelle, "Gauge theories of gravity and supergravity," In Ezawa, H. ( Ed.), Kamefuchi, S. ( Ed.): Progress In Quantum Field Theory, 57-81.

[8] M. Kaku, P. K. Townsend and P. van Nieuwenhuizen, "Gauge Theory of the Conformal and Superconformal Group,” Phys. Lett. 69B (1977) 304. doi:10.1016/0370-2693(77)90552-4

[9] E. S. Fradkin and A. A. Tseytlin, “Conformal Supergravity,” Phys. Rept. 119 (1985) 233. doi:10.1016/0370-1573(85)90138-3

[10] D. Z. Freedman and A. Van Proeyen "Supergravity," Cambridge University Press, 2012

[11] A. H. Chamseddine, "Supersymmetry and higher spin fields", PhD Thesis, (1976)

[12] A. H. Chamseddine and P. C. West, "Supergravity as a Gauge Theory of Supersymmetry," Nucl. Phys. B 129 (1977) 39. doi:10.1016/0550-3213(77)90018-9

[13] E. Witten, “(2+1)-Dimensional Gravity as an Exactly Soluble System,” Nucl. Phys. B 311 (1988) 46.

[14] A. H. Chamseddine, "Deforming Einstein's gravity," Phys. Lett. B 504 (2001) 33 doi:10.1016/S0370-2693(01)00272-6 [hep-th/0009153].

[15] A. H. Chamseddine, "SL(2,C) gravity with complex vierbein and its noncommutative extension," Phys. Rev. D 69 (2004) 024015 doi:10.1103/PhysRevD.69.024015 [hep-th/0309166].

[16] P. Aschieri and L. Castellani, "Noncommutative D=4 gravity coupled to fermions," JHEP 0906 (2009) 086 doi:10.1088/1126-6708/2009/06/086 [arXiv:0902.3817 [hep-th]].

[17] P. Aschieri and L. Castellani, "Noncommutative supergravity in D=3 and D=4," JHEP 0906 (2009) 087 doi:10.1088/1126-6708/2009/06/087 [arXiv:0902.3823 [hep-th]].

[18] M. Dimitrijević Ćirić, B. Nikolić and V. Radovanović, "Noncommutative $S O(2,3)$ „ gravity: Noncommutativity as a source of curvature and torsion," Phys. Rev. D 96 (2017) no.6, 064029 doi:10.1103/PhysRevD.96.064029 [arXiv:1612.00768 [hep-th]].

[19] S. Cacciatori, D. Klemm, L. Martucci and D. Zanon, "Noncommutative Einstein-AdS gravity in three-dimensions,” Phys. Lett. B 536 (2002) 101 doi:10.1016/S0370-2693(02)01823-3 [hep-th/0201103].

[20] S. Cacciatori, A. H. Chamseddine, D. Klemm, L. Martucci, W. A. Sabra and D. Zanon, "Noncommutative gravity in two dimensions," Class. Quant. Grav. 19 (2002) 4029 doi:10.1088/0264-9381/19/15/310 [hep-th/0203038].

[21] P. Aschieri and L. Castellani, "Noncommutative Chern-Simons gauge and gravity theories and their geometric Seiberg-Witten map,” JHEP 1411 (2014) 103 doi:10.1007/JHEP11(2014)103 [arXiv:1406.4896 [hep-th]].

[22] M. Banados, O. Chandia, N. E. Grandi, F. A. Schaposnik and G. A. Silva, "Three-dimensional noncommutative gravity,” Phys. Rev. D 64 (2001) 084012 doi:10.1103/PhysRevD.64.084012 [hep-th/0104264].

[23] N. Seiberg and E. Witten, "String theory and noncommutative geometry," JHEP 9909 (1999) 032 doi:10.1088/1126-6708/1999/09/032 [hep-th/9908142].

[24] J. Madore, Class. Quant. Grav. 9 (1992) 69. doi:10.1088/0264-9381/9/1/008 G. Manolakos and G. Zoupanos, Phys. Part. Nucl. Lett. 14 (2017) no.2, 322. doi:10.1134/S1547477117020194 
[25] G. Fiore and F. Pisacane, Lett Math Phys (2020) doi:10.1007/s11005-020-01263-3 [arXiv:1906.01881 [math-ph]].

G. Fiore and F. Pisacane, PoS CORFU 2017 (2018) 184 doi:10.22323/1.318.0184 [arXiv:1807.09053 [math-ph]].

G. Fiore and F. Pisacane, J. Geom. Phys. 132 (2018) 423 doi:10.1016/j.geomphys.2018.07.001 [arXiv:1709.04807 [math-ph]].

[26] H. Steinacker, "Emergent Geometry and Gravity from Matrix Models: an Introduction," Class. Quant. Grav. 27 (2010) 133001 doi:10.1088/0264-9381/27/13/133001 [arXiv:1003.4134 [hep-th]].

[27] V. P. Nair, "Gravitational fields on a noncommutative space," Nucl. Phys. B 651 (2003) 313 doi:10.1016/S0550-3213(02)01061-1 [hep-th/0112114].

[28] Y. Abe and V. P. Nair, "Noncommutative gravity: Fuzzy sphere and others," Phys. Rev. D 68 (2003) 025002 doi:10.1103/PhysRevD.68.025002 [hep-th/0212270].

[29] P. Valtancoli, “Gravity on a fuzzy sphere,” Int. J. Mod. Phys. A 19 (2004) 361 doi:10.1142/S0217751X04017598 [hep-th/0306065].

[30] V. P. Nair, "The Chern-Simons one-form and gravity on a fuzzy space," Nucl. Phys. B 750 (2006) 321 doi:10.1016/j.nuclphysb.2006.06.009 [hep-th/0605008].

[31] M. Burić, T. Grammatikopoulos, J. Madore and G. Zoupanos, "Gravity and the structure of noncommutative algebras," JHEP 0604 (2006) 054 doi:10.1088/1126-6708/2006/04/054 [hep-th/0603044].

[32] M. Burić, J. Madore and G. Zoupanos, "WKB Approximation in Noncommutative Gravity," SIGMA 3 (2007) 125 doi:10.3842/SIGMA.2007.125 [arXiv:0712.4024 [hep-th]].

[33] M. Burić, J. Madore and G. Zoupanos, “The Energy-momentum of a Poisson structure," Eur. Phys. J. C 55 (2008) 489 doi:10.1140/epjc/s10052-008-0602-x [arXiv:0709.3159 [hep-th]].

[34] P. Aschieri, J. Madore, P. Manousselis and G. Zoupanos, "Dimensional reduction over fuzzy coset spaces," JHEP 0404 (2004) 034 doi:10.1088/1126-6708/2004/04/034 [hep-th/0310072]; "Unified theories from fuzzy extra dimensions," ibid, Fortsch. Phys. 52 (2004) 718 doi:10.1002/prop.200410168 [hep-th/0401200]; ibid, hep-th/0503039.

[35] H. S. Snyder, “Quantized space-time," Phys. Rev. 71 (1947) 38. doi:10.1103/PhysRev.71.38

[36] C. N. Yang, “On quantized space-time,” Phys. Rev. 72 (1947) 874. doi:10.1103/PhysRev.72.874

[37] J. Heckman and H. Verlinde, “Covariant non-commutative space?time,” Nucl. Phys. B 894 (2015) 58 [arXiv:1401.1810 [hep-th]].

[38] A. Chatzistavrakidis, L. Jonke, D. Jurman, G. Manolakos, P. Manousselis and G. Zoupanos, "Noncommutative Gauge Theory and Gravity in Three Dimensions," Fortsch. Phys. 66 (2018) no.8-9, 1800047 doi:10.1002/prop.201800047 [arXiv:1802.07550 [hep-th]].

[39] D. Jurman, G. Manolakos, P. Manousselis and G. Zoupanos, "Gravity as a Gauge Theory on Three-Dimensional Noncommutative spaces," PoS CORFU 2017 (2018) 162 doi:10.22323/1.318.0162 [arXiv:1809.03879 [gr-qc]].

[40] G. Manolakos and G. Zoupanos, "Non-commutativity in Unified Theories and Gravity," Springer Proc. Math. Stat. 263 (2017) 177 doi:10.1007/978-981-13-2715-5-10 [arXiv:1809.02954 [hep-th]].

[41] A. B. Hammou, M. Lagraa and M. M. Sheikh-Jabbari, "Coherent state induced star product on $\mathrm{R}^{* * 3}$ (lambda) and the fuzzy sphere," Phys. Rev. D 66 (2002) 025025 doi:10.1103/PhysRevD.66.025025 [hep-th/0110291]. 
[42] P. Vitale, "Noncommutative field theory on $\mathbb{R}_{\lambda}^{3}$," Fortsch. Phys. 62 (2014) 825 doi:10.1002/prop.201400037 [arXiv:1406.1372 [hep-th]].

[43] S. Kováčik and P. Prešnajder, "The velocity operator in quantum mechanics in noncommutative space,” J. Math. Phys. 54 (2013) 102103 doi:10.1063/1.4826355 [arXiv:1309.4592 [math-ph]].

[44] D. Jurman and H. Steinacker, "2D fuzzy Anti-de Sitter space from matrix models," JHEP 1401 (2014) 100 doi:10.1007/JHEP01(2014)100 [arXiv:1309.1598 [hep-th]].

[45] G. Manolakos, P. Manousselis and G. Zoupanos, "Four-dimensional Gravity on a Covariant Noncommutative Space," arXiv:1902.10922 [hep-th]; G. Manolakos, P. Manousselis and G. Zoupanos, "Gauge Theories: From Kaluza-Klein to noncommutative gravity theories," Symmetry 11 (2019) no.7, 856. doi:10.3390/sym11070856; G. Manolakos, P. Manousselis and G. Zoupanos, "Noncommutative Gauge Theories and Gravity," PoS CORFU 2018 (2019) 096 doi:10.22323/1.347.0096 [arXiv:1907.06280 [hep-th]].

[46] A. H. Chamseddine, "Invariant actions for noncommutative gravity," J. Math. Phys. 44 (2003) 2534 doi:10.1063/1.1572199 [hep-th/0202137].

[47] L. F. Li, "Group Theory of the Spontaneously Broken Gauge Symmetries," Phys. Rev. D 9 (1974) 1723. doi:10.1103/PhysRevD.9.1723

[48] M. Burić and J. Madore, "Noncommutative de Sitter and FRW spaces," Eur. Phys. J. C 75 (2015) no.10, 502 doi:10.1140/epjc/s10052-015-3729-6 [arXiv:1508.06058 [hep-th]].

[49] M. Sperling and H. C. Steinacker, "Covariant 4-dimensional fuzzy spheres, matrix models and higher spin,” J. Phys. A 50 (2017) no.37, 375202 doi:10.1088/1751-8121/aa8295 [arXiv:1704.02863 [hep-th]].

[50] Y. Kimura, "Noncommutative gauge theory on fuzzy four sphere and matrix model," Nucl. Phys. B 637 (2002) 177 doi:10.1016/S0550-3213(02)00469-8 [hep-th/0204256].

[51] M. Burić, D. Latas and L. Nenadović, "Fuzzy de Sitter Space," Eur. Phys. G C78 (2018) No. 11,953 arXiv: 1709.05158 [hep-th].

[52] H. C. Steinacker, "Emergent gravity on covariant quantum spaces in the IKKT model," JHEP 1612 (2016) 156 doi:10.1007/JHEP12(2016)156 [arXiv:1606.00769 [hep-th]].

[53] J. Madore, S. Schraml, P. Schupp and J. Wess, "Gauge theory on noncommutative spaces," Eur. Phys. J. C 16 (2000) 161 doi:10.1007/s100520050012 [hep-th/0001203].

[54] A. Barut, "From Heisenberg algebra to Conformal Dynamical Group ' ' in A. Barut, H. D. Doener (Eds) "Conformal Groups and related Symmetries.Physical Results and Mathematical Backgrounf" Lecture Notes in Physics, Springer-Verlag 1985

[55] M. B. Green, J. H. Schwarz and E. Witten, "Superstring Theory. Vol. 2: Loop Amplitudes, Anomalies And Phenomenology,” Cambridge, Uk: Univ. Pr. ( 1987) 596 P. ( Cambridge Monographs On Mathematical Physics) 